\title{
Efectos de la suplementación de la L-carnitina sobre la composición corporal de ratas entrenadas y sedentarias
}

\section{Effects of supplementation of L-carnitine on the body composition of trained and sedentary rats}

\author{
R. GÓMEZ1, M. DE ARRUDA ${ }^{1}$, F. BORGES ${ }^{2}$, M.A. COSSIO ${ }^{1}$. \\ ${ }^{1}$ Departamento de Ciencias del Deporte, FEF, Universidad de Campinas, UNICAMP, Brasil.
}

${ }^{2}$ Universidad de Campinas, Departamento de Fisiologia.

\begin{abstract}
Resumen
El objetivo del presente estudio es determinar el efecto de la suplementación de L-carnitina sobre la composición corporal de ratas entrenadas y sedentarias. Fueron seleccionadas de forma aleatoria 12 ratas machos Wistar, de 12 semanas de vida, cuyo peso osciló entre 350 a 380 gramos, constituyéndose cuatro grupos de animales: sedentario no suplementado (SNS), sedentario suplementado (SS), entrenado no suplementado (ENS) y entrenado suplementado (ES). El entrenamiento fue realizado con sesiones de carrera en la estera ergométrica, con duración de una hora y con suplementación oral crónica de L-Carnitina, ambos protocolos con una duración de 4 semanas. La composición corporal fue evaluada a través de ecuaciones de predicción para determinar la masa libre de grasa $(M L G)$ y masa de grasa (MG). Los resultados muestran que el entrenamiento físico disminuye los valores de la MLG y MG en el grupo ENS, y que la administración de la L-carnitina en forma conjunta con el entrenamiento físico revierte parcialmente la reducción de la MLG en el grupo ES. Finalmente se concluye que el ejercicio físico debe ser realizado juntamente con la suplementación de L-carnitina, permitiendo una disminución de la MG y mantener los niveles de MLG en ratas en proceso de crecimiento.
\end{abstract}

Palabras clave: Composición corporal, entrenamiento, suplementación.

\begin{abstract}
The aim of this study is to determine the effect of L-carnitine supplementation on body composition of trained and sedentary rats. Were randomly selected 12 male Wistar rats of 12 weeks of age, whose weight ranged between 350 to 380 grams, four groups of animals: sedentary non-supplemented (SNS), sedentary supplemented (SS), trained not supplemented (ENS) trained and supplemented (ES). The training was done with sessions running on a treadmill, lasting an hour and chronic oral supplementation of L-carnitine, both protocols with a duration of 4 weeks. Body composition was assessed by prediction equations to determine fat-free (FFM) and fat mass (FM). The results show that physical training decreases the values of FFM and FM in the ENS group, and that the administration of L-carnitine together with physical training partially reverses the reduction of MLG in ES. Finally concluded that physical exercise should be conducted jointly with the supplementation of L-carnitine, allowing a reduction in the $M G$ and maintain levels in rats $M L G$ growth process.
\end{abstract}

Keywords: Body composition, training, supplementation.

Correspondencia:

Rossana Gómez Campos

Universidad Estadual de Campinas, Faculdade de Educação Física.

Av., Erico veríssimo 701, Cidade Universitária - 13083-851.

Caixa Postal 6134. Campinas, São Paulo, Brasil

E-mail: rossanagomez_c@hotmail.com 


\section{Introducción}

La composición corporal se caracteriza como una variable cineantropométrica, que es parte de una evaluación funcional [1], a través de la grasa corporal subcutanea de atletas y no atletas. En ese sentido, se refiere al estudio de la cantidad y proporción de los principales componentes estructurales del organismo, através del fraccionamento del peso corporal $[2,3,4]$. Su evaluación se basa en la separación del peso corporal total en diferentes compartimentos, cuya suma es igual a la masa corporal total [5]. Los valores de la composición corporal pueden diferenciar a los seres humanos en relación a la salud y al rendimiento físico, debido a que la distribución de la grasa corporal es un importante predictor de la morbidad y mortalidad [6], existiendo una correlación inversa entre una cantidad elevada de grasa corporal con la capacidad física [7], de esa manera según Heyward y Stolarczyk [8], son varias las aplicaciones que se realizan conociendo los valores de la composición corporal.

Por otro lado, la suplementación alimentar esta siendo usada por atletas y por personas que practican actividad física, con el objetivo de mejorar el rendimiento $\mathrm{y} / \mathrm{o}$ el gano de salud y forma física [9. En ese sentido, la carnitina al ser un componente vital en el metabolismo de los lípidos [10], cumpliendo la función de actuar en las reacciones transferidoras de ácidos grasos libres de cadena larga del citosol para las mitocondrias, bajo la forma de acilcarnitina, facilitanto su oxidación y generación de ATP (adenosina trifosfato) [11], podría ser usada para incrementar el rendimiento atlético y mantener una carga de trabajo $[12,13]$; además de presentar aplicaciones en la reducción del contenido lipidico del tejido animal [14], y en algunos casos podría permitir la disminución de la masa de grasa, por lo que ha despertado la atención de los investigadores para las aplicaciones clínicas y experimentales asociadas a su deficiencia en diversos procesos patológicos, bien como a los efectos de su suplementación en la práctica de actividad física. De esta manera, el presente estudio tuvo por objetivo determinar el efecto de la suplementación de L-carnitina sobre la composición corporal de ratas entrenadas y sedentarias.

\section{Material y métodos}

\section{Muestra y tipo de investigación}

El estudio es de tipo experimental [15], donde fueron seleccionados ratas machos adultos de forma aleatoria de linage Wistar con 12 semanas de vida, cuyo peso osciló entre 350 a 380 gramos, provenientes del Bioterio del departamento de Farmacologia de la UNICAMP. Los animales fueron mantenidos en cajas colectivas con cinco animales cada una, en ciclos de claro/escuro (12/12 h), recibiendo ración padrón (Labina, Purina) y agua ad libitum.

Fueron constituidos, 4 grupos de animales $(\mathrm{n}=5)$ : sedentario suplementado con L-carnitina (SS), sedentario no suplementado (SNS), entrenado suplementado con L-carnitina (ES) y entrenado no suplementado (ENS).

\section{Evaluación del peso corporal}

Para la evaluación del peso corporal $(\mathrm{g})$ de las ratas se utilizó una balanza analítica de marca Scaltec modelo SAC-62, con una precisión de (10-4gramas). El procedimento consistió en colocar a los animales en un frasco ligero sobre la balanza con el objetivo de evaluar el peso corporal en gramos $(\mathrm{g})$. Ese procedimento fue repetido dos veces durante la evaluación con la intención de verificar el error técnico de medida ETM intra-evaluador $(2 / 2 n)$, determinándose también la reproductibilidad del peso a través del coeficiente de Pearson (r). El peso corporal fue evaluado cada semana durante el tiempo de duración del estudio.

\section{Evaluación del peso metabólico}

Para la evaluación del peso metabólico (PM) (g) de ratas machos, se utilizó la ecuación sugerida por Perez-García [16], basada en el peso corporal, a través de la fórmula: $\mathrm{PM}=\mathrm{PV}$ x 0,75

\section{Suplementación}

Los animales fueron suplementados vía oral durante 4 semanas, con una solución conteniendo aproximadamente $1 \mathrm{mg} / \mathrm{ml}$ de L-car [17], lo que representa una dosis aproximada de $0.2 \mathrm{~g} / \mathrm{kg}$ por dia. Esta dosis representa aproximadamente el doble del consumo diario de Carnitina [18] y mantiene los niveles plasmáticos de carnitina en aproximadamente 3 veces por encima de los niveles observados en animales control.

\section{Entrenamientos de las ratas}

Las sesiones de entrenamiento fueron realizadas en una estera ergométrica eléctrica, con bayas individuales de $0,70 \mathrm{~m}$ de ancho, $0,45 \mathrm{~m}$ de altura y $1,35 \mathrm{~m}$ de largo. Una semana antes de iniciar el entrenamiento, todos los animales fueron sometidos a un periodo de adaptación a la estera que consistió en mantener los animales en la estera en 
velocidad variando entre 0,3 en el primer día, hasta $0,6 \mathrm{Km} / \mathrm{h}$ en el quinto día de la semana, aumentando progresivamente el tiempo en la duración de las sesiones hasta que los animales consiguieron permanecer corriendo en la estera por 60 minutos.

El entrenamiento físico se realizó con una duración de 60 minutos, durante 4 semanas, iniciando a una velocidad de $0,6 \mathrm{Km} / \mathrm{h}$ en la primera sesión, aumentando progresivamente conforme la evolución del grupo de animales, hasta alcanzar la velocidad final de $1,2 \mathrm{Km} / \mathrm{h}$ a partir de la segunda semana de entrenamiento. Esta intensidad de ejercicio corresponde a la velocidad en que los animales alcanzan el máximo estado estable de lactato, lo que corresponde a una intensidad aerobia de ejercicio.

\section{Evaluación de la composición corporal}

Para la evaluación de la composición corporal se utilizó las ecuaciones propuestas por CossioBolanos, et.al.[19], basadas en mediciones antropométricas y consideradas como un método doblemente indirecto. Donde a partir de esa propuesta se calculo la masa libre de grasa (MLG) y masa de grasa (MG), respectivamente. La diferencia de la sumatoria de esos componentes, sustraida del total viene a constituir la masa residual.

\section{Análisis estadístico}

Los resultados del presente estudio fueron analizados a través de la estadística descriptica de media (X) y error estándar (EEM) y para determinar las diferencias estadísticas entre los grupos se utilizó la test t, y la prueba de especificidad de Tukey $(\mathrm{p}<0,001)$

\section{Resultados}

La tabla 2 muestra los resultados del error técnico de medida ETM intra-evaluador del peso (g) de las ratas machos de los 4 grupos, donde esos resultados muestran valores bajos $(0,55$ a 0,94$)$ de ETM, siendo aceptables y menores al $1 \%$. Por otro lado, el coeficiente de correlación de Pearson se utilizó para determinar la capacidad de reproductibilidad de la medida, mostrando valores altamente significativos $(0,99)(\mathrm{p}<0,001)$.

\begin{tabular}{|lll|}
\hline Compartimentos & Ecuación & $\mathbf{R}^{\mathbf{2}}$ \\
\hline Masa libre de grasa (MLG) & $\mathrm{MLG}=20,1+(0,48 *$ peso total) & 0,944 \\
Masa de grasa (MG) & $\mathrm{MG}=-32,2+(0,28 *$ peso total) & 0,721 \\
\hline
\end{tabular}

Tabla 1. Ecuaciones usadas para el cálculo de los dos componentes corporales según [19].

\begin{tabular}{|lllll|}
\hline Grupos & N & ETM & R & P \\
\hline SS & 10 & 0,894427 & 0,998953 & 0,0009 \\
SNS & 10 & 0,948683 & 0,999261 & 0,0007 \\
TS & 5 & 0,632456 & 0,99932 & 0,0006 \\
TNS & 5 & 0,547723 & 0,99977 & 0,0002 \\
\hline
\end{tabular}

Tabla 2. Error técnico de la medida intra-evaluador (ETM) y coeficiente de reproductibilidad (r) del peso corporal (g) de los 4 grupos estudiados. 
La tabla 3 muestra las características de la población estudiada, donde se puede observar los valores medios $(\mathrm{X})$ y error standar (EEM) del peso total inicial (g), el peso final (g) y el peso metabólico (g), de los grupos SS, SNS, ES y ENS, así como el gano de peso corporal en gramos y porcentaje, y el peso del músculo gastronemio (g) al final de las 4 semanas de estudio.

Se observa diferencias significativas $(p<0,001)$ en los grupos SNS, SS y ES, pero no en el grupo ENS. Estos resultados evidencian que el entrenamiento aeróbico produce menor gano de peso corporal, por consecuencia una disminución en el crecimiento somático en ratas en proceso de cre- cimiento, siendo que el músculo gastronemio del grupo ENS $(2,7 \pm 0.2)$ presenta valores inferiores en relación a los otros grupos.

Por otro lado, cuando se comparó el peso final, como se observa en la figura 1 , hubo diferencias significativas $(\mathrm{p}<0,005)$ entre los grupos (SNS y ENS) y (SS y ES), debido a que el entrenamiento aeróbico (ENS) reduce significativamente el peso total en relación al grupo que tuvo entrenamiento y suplementación de L-carnitina (ES). Sin embargo, no hubo diferencias significativas entre los grupos (SNS y SS) y (ENS y ES), debido a que la suplementación de la L-carnitina durante las 4 semanas no fue suficiente para modificar el peso corporal de las ratas.

\begin{tabular}{|lllll|}
\hline Grupos & SNS & SS & ENS & ES \\
\hline $\mathrm{N}$ & 10 & 10 & 5 & 5 \\
Peso inicial (g) & $353 \pm 12$ & $353 \pm 12$ & $358 \pm 14$ & $356 \pm 17$ \\
Peso final (g) & $456 \pm 7^{\mathrm{a}}$ & $435 \pm 9^{\mathrm{a}}$ & $375 \pm 17^{\mathrm{bc}}$ & $401 \pm 9^{\mathrm{abc}}$ \\
Peso metabólico (g) & $342 \pm 5$ & $327 \pm 7$ & $281 \pm 13^{\mathrm{b}}$ & $301 \pm 7^{\mathrm{b}}$ \\
Gano do peso (g) & $103 \pm 13$ & $83 \pm 12$ & $17 \pm 12$ & $45 \pm 15$ \\
Variación del peso (\%) & 29.2 & 23.6 & 4.9 & 12.8 \\
$\begin{array}{l}\text { Peso del músculo gastronemio } \\
\text { (g) }\end{array}$ & $3,0 \pm 0.1$ & $3,2 \pm 0.1$ & $2,7 \pm 0.2$ & $3,1 \pm 0.2$ \\
\hline $\begin{array}{l}\text { Los datos representan las medias } \pm \text { error standard para cada grupo de animales. } \\
\text { (a) indica diferencia estadística entre los valores inicial y final para cada grupo. } \\
\text { (b) indica diferencia estadística en relación al grupo SNS. } \\
\text { (c) indica diferencia estadística en relación al grupo SS. } \\
\text { (d) indica diferencia estadística entre los grupos ES y ENS }\end{array}$ & & \\
\hline
\end{tabular}

Tabla 3. Caracterización de la muestra según [19].

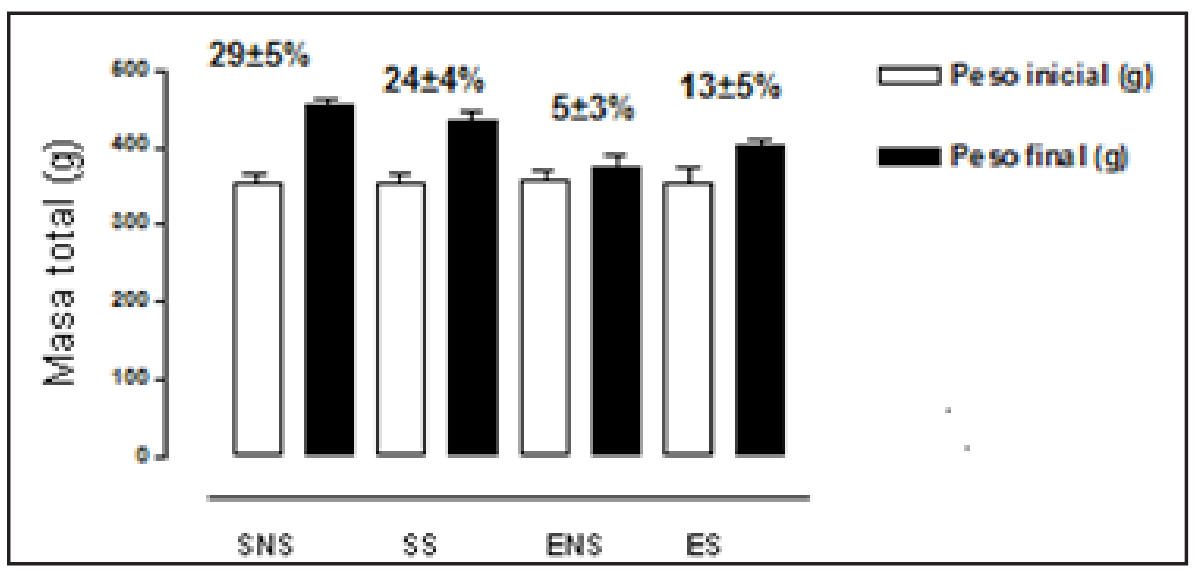

Figura 1. Peso corporal inicial y final (g) dos grupos. 
La figura 2 muestra los valores medios (X) y error standar (EEM) de la masa libre de grasa (MLG), masa de grasa (MG) em gramos de los grupos SNS, SS, ENS y ES, obtenidos a través de las ecuaciones de predicción usando como variable independiente el peso corporal.

El fraccionamiento de los componentes corporales, muestra diferencias significativas entre los grupos (SNS y ENS), (SNS y ES), (SS y ENS) y (SS y ES), una vez que la MLG fue afectada significativamente por el entrenamiento aerobico en el grupo ENS, reduciendo la MLG en mayor proporción en relación al grupo que tuvo entrenamiento y suplementación (ES). Sin embargo, no hubo diferencias significativas entre los grupos (SNS y SS) y (ENS y ES) debido a que la suplementación de la L-carnitina no tuvo efecto sobre la MLG.

En relación a la MG los resultados permiten observar diferencias significativas entre los grupos (SNS y ENS), (SNS y ES), (SS y ENS), (ENS y ES) y (SS y ES). Debido a que el entrenamiento aerobico (ENS) permite reducir significativameante la $\mathrm{MG}$, siendo esa proporción mayor em relación al grupo que tuvo entrenamiento y suplementación (ES). Sin embargo, no fueron encontrados diferencias significativas entre los grupos (SNS y SS), por lo que, a L-carnitina no fue capaz de reducir la MG después de las 4 semanas de estudio.

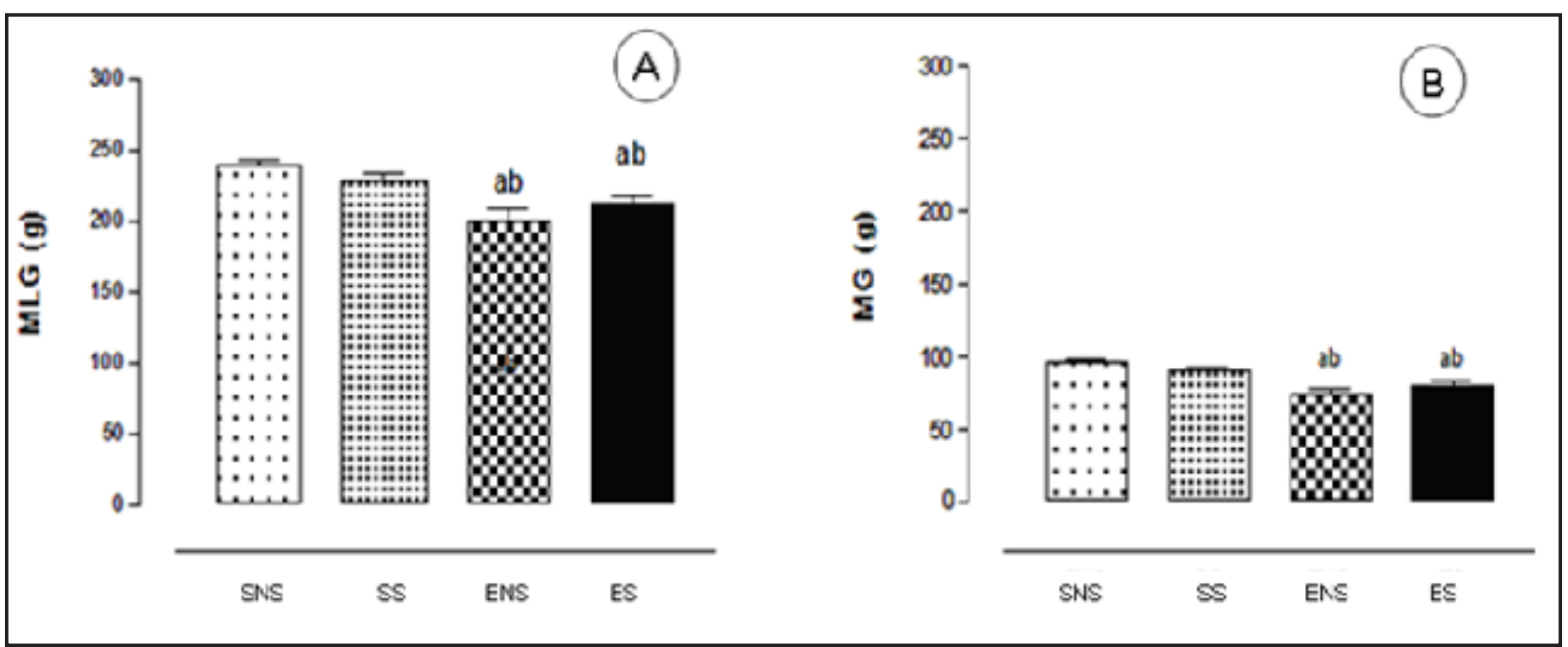

Figura 2. Masa libre de grasa (A), masa de grasa (B) de los grupos estudiados. (a) $\mathrm{P}<0.05$, comparado al grupo SNS; (b) $\mathrm{P}<0.05$ comparado al grupo SS.

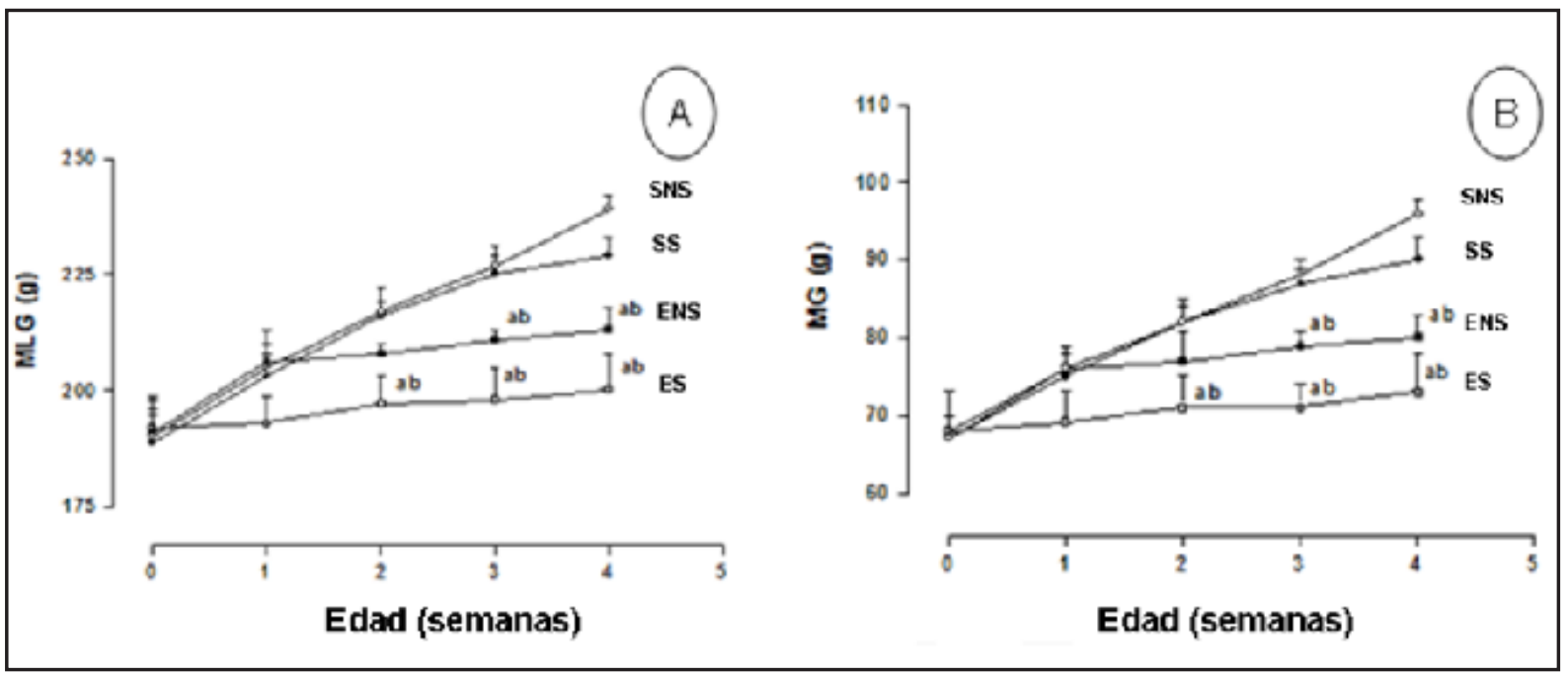

Figura 3. Evolución de la MLG (A) y MG (B) durante las 4 semanas de tratamiento. (a) diferencia estadística en relación al grupo SNS; (b) indica diferencia estadística en relación al grupo SS. 
La figura 3 muestra que la evolución de la MLG en el grupo sedentario no suplementado, es creciente durante las 4 semanas de estudio. Resultados similares son observados en el grupo SS, mostrando que la suplementación con L-carnitina no afecta el desarrollo de la MLG en ratos sedentarios. Por otro lado, el entreinamento físico promueve um menor aumento de la MLG, y a partir de la segunda semana de estudio, esa diferencia ES fue más acentuada en comparación al grupo de animales sedentarios.

El tratamiento con L-carnitina asociado al ejercicio físico retarda esa reducción de la MLG, que fue significativa a partir de la tercera semana de estudio cuando fue comparado al grupo SNS y SS. Así también, la evolución de la MG fue creciente en el grupo SNS durante las 4 semanas de estudio. La L-carnitina no afectó la evolución de la MG en animales sedentarios (SS). Por otro lado, el entrenamiento físico promovió una reducción en la evolución de la MG a partir de la segunda semana de estudio, en cuanto que la suplementación com L-carnitina asociada al ejercício físico promovió reducción en la evolución de la MG a partir de la tercera semana de estudio.

\section{Discusión}

La carnitina es un constituyente natural del organismo y facilita el transporte de la grasa para la $\beta$-oxidación [20], por lo que es necesario un adecuado estoque de carnitina [21]. Nuestros resultados muestran que la suplementación con Lcarnitina no afectó el gano de peso corporal de los animales y previnió parcialmente la reducción del peso inducida por el ejercicio físico, siendo que el gano de peso fue significativamente menor en animales sometidos a entrenamiento físico. Los efectos de la L-carnitina sobre la composición corporal son controversos, un estudio previo demostró que la L-carnitina no afectaba la estimulación de la lipólisis de forma de aumentar la cantidad de grasa como substrato para el músculo de ratas entrenadas [22]. Eso podría explicar la ausencia de un efecto pronunciado de la L-carnitina sobre la MG observada en nuestro estudio.

Durante el estudio, la evolución de la MLG y MG muestra que en los animales sometidos a entrenamiento físico, ocurrio una reducción en la evolución de los dos componentes evaluados, efecto que fue parcialmente revertido por la suplementación realizada en forma conjunta con el entrenamiento físico. En ese sentido, cabe destacar que el entrenamiento junto com la suplemen- tación, serian factores importantes para reducir la MG, debido a que, sólo el entrenamiento aeróbico, además de reducir la MG también reduce la MLG, por lo que, podría producir un desequilibrio en la composición corporal de las ratas. Estos resultados sugieren que el entrenamiento aeróbico debería ser realizado en forma conjunta con el consumo de L-carnitina, evitando una disminución drástica de la MLG, pero si la disminución gradual de la $\mathrm{MG}$, respectivamente.

Así mismo, es ampliamente conocido que los ejercicios físicos de larga duración de baja y moderada intensidad utilizan predominantemente ácidos grasos como combustible energético, en función del aumento de la demanda energética y metabólica requerida por el ejercicio físico, lo que lleva a la reducción de la MG [23-26]. Sin embargo, en nuestro estudio, el menor gano de la MLG en animales sometidos al entrenamiento físico puede estar asociado a una mayor utilización de proteinas como fuente energética durante la actividad física. Estudios muestran que la tasa de oxidación de los aminoácidos durante el ejercicio es mayor que en reposo y que los aminoacidos son utilizados en gran extensión como fuente de energia durante el ejercicio físico [27-29], por esta razón, se afirma que la demanda proteica para individuos entrenados sea mayor que la cantidad diaria recomendada para individuos sedentarios, especialmente a los practicantes de ejercicios prolongados [30].

Finalmente, es necesario destacar que para la determinación de la MLG y MG, fue utilizado un método indirecto (Ecuación de predicción), que podría tener algunas limitaciones en el grado de validez y exactitud. Por tanto, es necesario continuar con investigaciones que objetivisen los reales efectos del entrenamiento aeróbico y la suplementación de la L-carnitina sobre la composición corporal de ratas en proceso de crecimiento e inclusive en ratas adultos.

\section{Conclusión}

A través de los resultados obtenidos en el presente estudio, se puede concluir que el entrenamiento aeróbico durante 4 semanas en ratas en proceso de crecimiento, produce uma disminución de la masa libre de grasa y masa de grasa, así también la L-carnitina de forma independiente no produce efecto sobre los dos componentes, sin embargo el entrenamiento realizado en forma conjunta con la suplementación de L-carnitina, reducen la masa de grasa y mantienen niveles aceptables de 
masa libre de grasa, previniendo parcialmente el efecto inducido por el ejercicio físico, debido a que el ejercicio físico de forma independiente diminuye aún más la masa libre de grasa y podria producir uma descompensación en la composición corporal de las ratas.

\section{Referencias}

1. Ribeiro JP, Luzardo A, De Rose EH. Potencia anaeróbica em indivíduos treinados e não treinados. Revista brasileira de ciências do esporte 1980;1(3):11-15.

2. Malina RM, Bouchard C. Growth, maturation and physical activity. Champaign: Human Kinetics, 1991

3. Lopes AS, Pires-Neto CS. Composição corporal e equações preditivas da gordura em crianças e jovens. Rev. Brasileira Atividade física e saúde 1996;4(1):38-52.

4. Petroski EL. Antropometría, Técnicas e padronizações. Editora Pallotti, Porto Alegre, 1999.

5. McArcle K. Fisiología del ejercicio: Energía, nutrición, y desempeño motor. 4ta edicao, Editora Guanabara koogan, R.J. Brasil, 1998.

6. Gustat J. Relation of abdominal height to cardiovascular risk factors in young adults. Am J Epidemiol 2000;151(9):885-91.

7. Salem M, Amaral R, Montella E, Walz M, Nakashima G, Pueringer P, Reis C, Junior C, Concceição C. Desenvolvimento e validação de equações para a estimativa da porcentagem de gordura dos alunos do curso de instrutor da escola de educação Física do exército. Revista de Educação Física 2006;133:49-58.

8. Heyward VH, Stolarczyk LM. Avaliação da composição corporal aplicada. São Paulo: Manole, 1996.

9. Gomez MR, Tirapegui J. Relação de alguns suplementos nutricionais e o desempheno físico. Arch latinoam Nutri 2000;50:317-329.

10. Carter AL, Abney PO, Lapp FD. Byosintesis and metabolismo F-carnitine. J. Child. Neurol 1995:10:253-257.

11. Coelho C, Mota J, Bragança E, Burini R. Aplicações clínicas da suplementação de L-carnitina. Ver. Nutr., Campinas 2005;18(5):651-659.

12. Swart L, Rossouw J, Loots JM, Kruger MC. The effect of L-carnitine supplementation on plasma carnitine levels and various performance parameters of male marathon athletes. Nutrition Research 1997;17(3):405-414.

13. Lennon D, Stratman F, Shrago E. The effects of exercise training on carnitine status and physical work capacity in dialysis patients. Medicine and Science in Sports and exercise 1983;14:165.
14. Reda E, D'Iddio S, Nicolai R, Benatti P, Calvani $M$. The carnitine system and body composition. Acta Diabetol 2003;40:S106-S112.

15. Thomas J, Nelson J. Research Methods in Physical Activity. Champaign: Human Kinetics, 2002.

16. Perez García C, Diez Prieto M., García Partida P. Introducción a la experimentación animal. Universidad de León: España, 1999.

17. Malone JI, Cuthbertson DD, Malone MA, Schocken DD. Cardio-protective effects of carnitine in streptozotocin-induced diabetic rats. Cardiovasc Diabetol 2006;19(5):2.

18. Borum PR. Carnitine. Annual Review of Nutrition 1983;3:233-259.

19. Cossio-Bolaños MA, Gómez-Campos R, Rojas J, Flores H. Propuesta de ecuaciones para predecir la composición corporal de ratas machos wistar. An Fac med 2010;71(2):97-102.

20. Leibovitz B, Mueller J. Carnitine. J Opt Nutr 1993;2:90-109.

21. Brass EP. Supplemental carnitine and exercise. Am J. Clin Nutr 2000;72(2 suppl): 618-623.

22. Décombaz JE, Reffet B, Bloemhard Y. Effect of L-carnitine and stimulated lipolysis on muscle substrates in the exercising rat. Experientia 1990;46:457-458.

23. Coyle EF, Jeukendrup AE, Wagenmakers AJM, Saris WHM. Fatty acid oxidation is directly regulated by carbohydrate metabolism during exercise. Am J Physiol 1997;273(2):E268E275.

24. Curi R, Lagranha C, Rodriguez J, Pithon-Curi T, Lancha JR, Pellegriinotti I, Procopio J. Ciclo de Krebs como fator lilitante na utilziação de ácidos graxos durante o exercício aeróbico. Arquivos Brasileros de Endocrinologia \& metabología 2003;47(2):135-143.

25. Wolfe RR, Klein SS, Carraro F, Weber JM. Role of triglyceride-fatty acid cycle in controlling fat metabolism in humans during and after exercise. Am J Physiol 1990;258:E382-389.

26. Ronsen O, Kjeldsen-Kragh J, Haug E, Bahr R, Pedersern BK. Recovery time affects inmunoendocrine responses to a second bout of endurance exercise. Am J Physiol Nutrition Metabolism 2005;30(6):723-734.

27. Williams C. Nutritional aspects of exercise-induced fatigue. Proc Nutr Soc 1985;44:245-256.

28. Davis J. Carbohydrates, branched-chain amino acids, and endurance: the central fatigue hypothesis. Int J Sport Nutr 1995;5:S29-38.

29. Hood DA, Terjung RL. Amino acid metabolism during exercise and following endurance training. Sports Medicine 1990; 9:23-35.

30. Lemon PWR, Proctor DN. Protein intake and athletic performance. Sports Medicine 1991;12:313-325. 